\title{
Portrait of a year-old pandemic
}

\section{'Swine flu' isn't over yet, but it already holds lessons for the future.}

One year ago this month, the world watched with trepidation as a novel influenza A virus, to which the global population had little or no immunity, emerged in Mexico and the United States. In the weeks that followed, the H1N1 'swine flu' virus spread rapidly to countries worldwide, leading the World Health Organization (WHO) on 11 June 2009 to officially declare the first flu pandemic in more than 40 years. Nature looks at the lessons learnt from $\mathrm{H} 1 \mathrm{~N} 1$, and how they will help scientists and health authorities to handle the next flu pandemic.

\section{How severe has the pandemic been so far?} The 2009 pandemic was not the killer of 1918. "Most people were less likely to get infected than in previous pandemics, less likely to get sick if they did, and less likely to die if they got sick," says Marc Lipsitch, an epidemiologist at Harvard School of Public Health in Boston, Massachusetts. But with the pandemic still playing out, it may be years before we get a reliable estimate of how many deaths it has caused. Research published last month in the journal PLoS Currents Influenza, by Cécile Viboud of the US National Institutes of Health (NIH) in Bethesda, Maryland, and her colleagues, suggests that the first waves may have been more severe than is widely perceived ${ }^{1}$.

Viboud and her team tested different approaches for estimating flu mortality in the United States, with the most conservative estimates based on medical records that officially reported flu as the cause of death. This is likely to be an underestimate: many flu deaths are not recorded as such, with death often ascribed instead to an underlying condition, such as heart disease or diabetes.

This conservative approach estimated US pandemic flu deaths at around 7,500-12,000: less than half the number caused annually in the United States by seasonal influenza $\mathrm{H} 1 \mathrm{~N} 1$ and influenza B viruses. But this method also revealed that the number of life years lost was around a quarter more than usual because the 2009 pandemic deaths were skewed towards younger ages than seasonal flu (see 'Deaths and years

\section{DEATHS AND YEARS OF LIFE LOST FROM INFLUENZA \\ New estimates of the toll of the $2009 \mathrm{H} 1 \mathrm{~N} 1$ pandemic in the United States show that, in terms of 'years of life lost', it may have been comparable to earlier pandemics.}
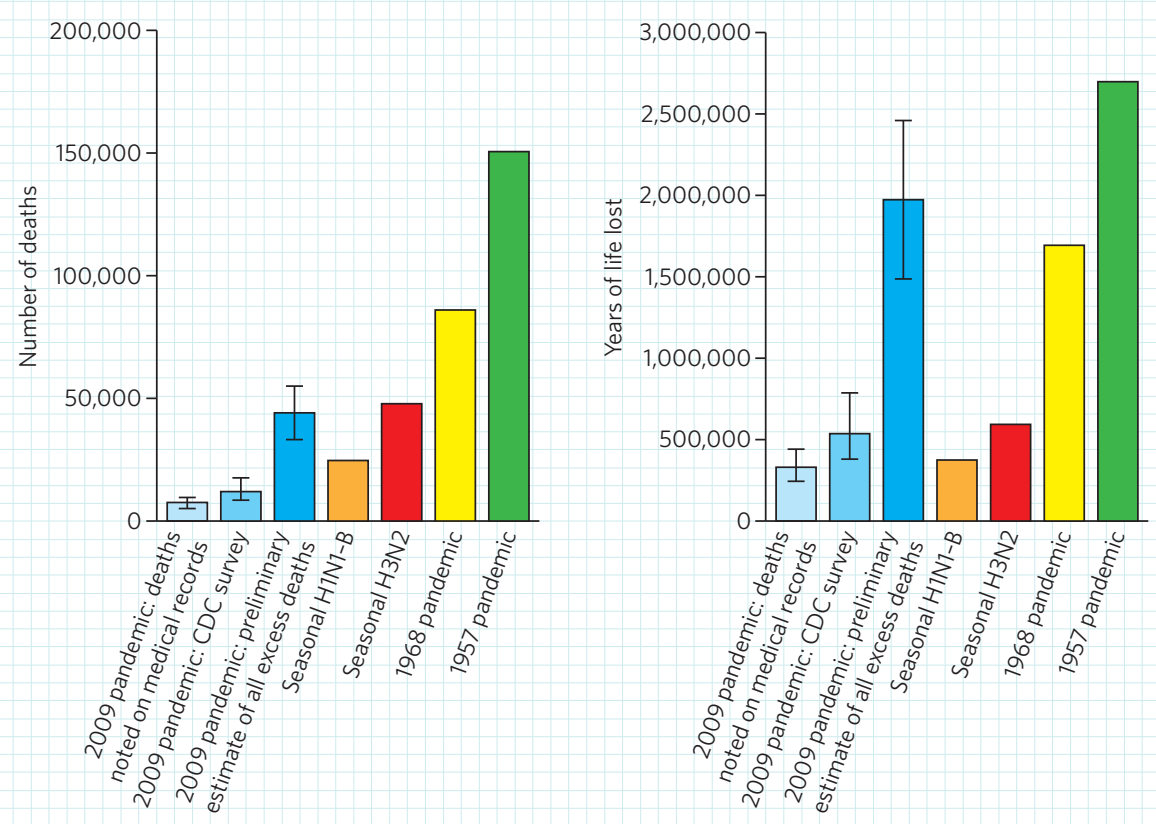

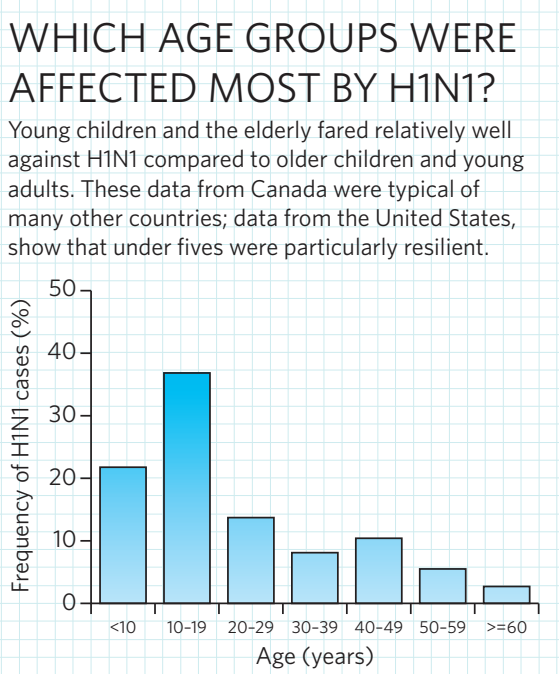

of life lost from influenza). Under a less conservative estimate, based on comparing overall mortality during the pandemic with mortality over the same period in previous years, excess deaths numbered 44,100, surpassing those of a typical flu season. Years of life lost were three to four times higher than a virulent $\mathrm{H} 3 \mathrm{~N} 2$ season and five times higher than years of life lost to seasonal $\mathrm{H} 1 \mathrm{~N} 1$ and $\mathrm{B}$ viruses - of the same order as the 1968 flu pandemic.

\section{Why were older people largely spared?}

Whereas seasonal flu hits mainly the very old and the very young, the 2009 pandemic was different in that those most affected were older children and young adults (see 'Which age groups were affected most by H1N1?'). Research published in January 2010 in the journal BMC Infectious Diseases reported that more than three-quarters of cases occurred in people younger than 30 , with a peak in the group aged 10-19 years ${ }^{2}$.

Seroprevalence studies, which monitor antibodies that react with the virus, suggest an explanation for this. Serum samples taken in England before the start of the pandemic show that older people had stronger antibody reactions to H1N1 than young people, probably as a result of previous exposure to strains with similarities to the new virus ${ }^{3}$. Because the pandemic virus outcompeted seasonal flu strains, the usual annual flu season never developed, so older people 


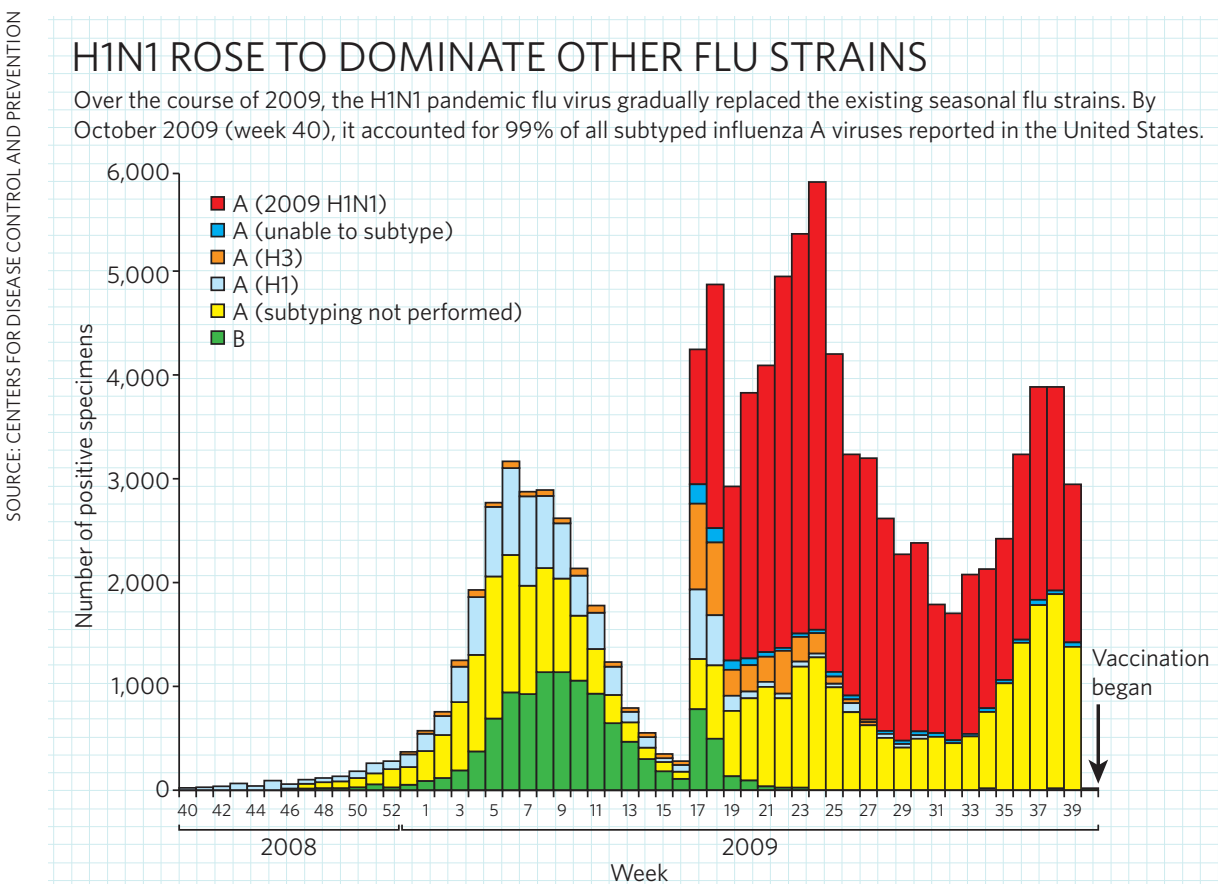

were spared a bad A/H3N2 flu season, which normally takes a heavy toll on the elderly.

\section{Will there be more waves of infection?}

Probably. In past pandemics, infection and illness have come in waves over a period of several years, and later waves are often more severe. "We are in a pandemic period of 2 to 5 years and must continue to keep our guard up," says Lone Simonsen, a flu expert at the Research and Policy for Infectious Disease Dynamics (RAPIDD) programme, a collaboration between the NIH Fogarty International Center and the science and technology directorate of the US Department of Homeland Security.

In the longer term, the $\mathrm{H} 1 \mathrm{~N} 1$ virus looks set to establish itself as the dominant seasonal flu strain. Already, almost all new flu cases currently being detected are pandemic H1N1 (see 'H1N1 rose to dominate other flu strains'), although some seasonal influenza $B$ virus continues to co-circulate, particularly in Asia. As more people acquire resistance to the pandemic virus, its virulence will settle to the level of seasonal flu, which causes new infections only when the genetics of the virus change annually.

This winter saw little flu overall in the Northern Hemisphere. Outbreaks of pandemic flu are currently occurring in some of the tropical zones of the Americas, west and east Africa and southeast Asia, in particular Thailand and Singapore, but at low levels. With winter approaching in the Southern Hemisphere, the question of whether a new pandemic wave will hit countries there will soon be answered.

\section{Have we learnt any lessons from the 2009 pandemic?}

H1N1 offered a stark reminder that current techniques for making a flu vaccine take too long: around six months from the identification of the new virus to production of any sizeable vaccine quantities. Substantial amounts of vaccine against the pandemic

virus became available only around last October, after the first wave had already passed during the winter in Australia (see 'Vaccines arrived too late') and in other Southern Hemisphere countries, and weeks into an autumn wave in most Northern Hemisphere countries.

Better surveillance is also needed. Although researchers could gain some idea of the progress of the pandemic from the number of cases diagnosed and from proxy figures - such as hospitalizations and the number of people reporting influenza-like illnesses - health agencies and scientists were generally slow to implement the gold standard: seroprevalence studies that measure levels of flu antibodies in blood serum (see Nature 462, 398-399; 2009). Part of the problem was logistical: at the start of the pandemic, most labs were overwhelmed with diagnostic samples that needed immediate processing to meet pressing public-health demands. But a lack of advance planning often hampered efforts as well.

Good seroprevalence data are crucial to making informed policy decisions. A key measurement of the severity of a pandemic is the case-fatality rate, or how often infection is fatal. Getting a handle on this requires precise estimates of how many people have been infected, but it was not until around last September - five months into the pandemic - that epidemiologists began to get such data.

Clinical research during the pandemic - studies of the best drug regimes, for example - also lagged. In several countries, such as the United Kingdom and Australia, medical research councils introduced calls for proposals at record speed. But compared with epidemiologists and virologists, clinical researchers were generally slow to respond, in part because many were tied up in the frontline pandemic response. Anne Kelso, director of the WHO Collaborating Centre for Reference and Research on Influenza in Melbourne, Australia, points to another problem: "Delays in obtaining ethical clearance was another impediment for some, with the result that the epidemic was almost over before some studies could begin."

Declan Butler

1. Viboud, C., Miller, M., Olson, D., Osterholm, M. \& Simonsen, L. PLoS Curr. Influenza, RRN1153 (2010). Available at go.nature.com/DSfm6h.

2. Reichert, T., Chowell, G., Nishiura, H.,

Christensen, R. A. \& McCullers, J. A. BMC Infect. Dis. 10, 5 (2010).

3. Miller, E. et al. Lancet 375, 1100-1108 (2010). 